\title{
A Faunistic List for the Superfamilies Papilionoidea, Geometroidea, Cossoidea, Lasiocampoidea, Bombycoidea, Drepanoidea, Noctuoidea and Pyraloidea (Lepidoptera) of Muğla Province, South-Western Turkey
}

\author{
Erdem SEVEN ${ }^{1^{*}}$, Nafiz YILMAZ ${ }^{2}$ : \\ ${ }^{1}$ Batman University, School of Tourism and Hotel Management, Batman, TURKEY \\ ${ }^{2}$ Batman University, Vocational High School (Retired Lecturer), Batman, TURKEY \\ *Corresponding Author: erdem seven@ hotmail.com
}

Received Date: 30.10 .2020

Accepted Date: 25.02 .2021

\section{Abstract}

Aim of study: The present study aimed to contribute Lepidoptera fauna of Muğla province and Turkey.

Area of study: Research was carried out in Muğla province, Ula district, Gökova, Akyaka Forest Camp.

Material and methods: Butterfly and moth samples were collected between in 2018-2020 with net and mercury vapour lamp. Specimens were arranged according to standard methods and diagnosed. Materials were stored at Entomology Laboratory of Batman University.

Main results: In total, 133 species were identified and, 31 of them are new discovered in Muğla province.

Highlights: Lepidoptera species of Muğla province, especially Geometroidea and Pyraloidea groups, have not been well studied. The results of the research contribute to the elimination of this deficiency. In addition, information on the vegetation of the study area is given for the first time. Eastern Pine Processionary Moth (Thaumetopoea wilkinsoni Tams, 1925), which is an oligofag defoliator in pine forests, has been observed with the high population density in the research area.

Keywords: Lepidoptera, Butterfly, Moth, Fauna, Muğla, Turkey

\section{Türkiye'nin Güneybatısındaki Muğla İlinin Papilionoidea,}

\section{Geometroidea, Cossoidea, Lasiocampoidea, Bombycoidea, Drepanoidea, Noctuoidea ve Pyraloidea (Lepidoptera) Üstfamilyaları için Faunistik Bir Liste}

Öz

Çalışmanın amacı: Bu çalışma, Muğla ili ve Türkiye Lepidoptera faunasına katkıda bulunmayı amaçlamaktadır.

Çalışma alanı: Araştırma Muğla ili, Ula ilçesi, Gökova, Akyaka Orman Kampı’nda yapılmıştır.

Materyal ve yöntem: Kelebek ve güve örnekleri 2018-2020 yılları arasında atrap ve civa buharlı lamba ile toplanmıştır. Örnekler standart yöntemlere göre düzenlenmiş ve teşhis edilmiştir. Materyaller, Batman Üniversitesi Entomoloji Laboratuvarı'nda korunmaktadır.

Temel sonuçlar: Toplamda 133 tür tespit edilmiş ve bunların 31'i Muğla ilinde yeni keşfedilmiştir.

Araştırma vurguları: Muğla ilinin Lepidoptera türleri, özellikle Geometroidea ve Pyraloidea grupları, iyi çalışılmamıştır. Araştırma sonuçları bu eksikliğin giderilmesine katkıda bulunmaktadır. Ayrıca çalışma alanının bitki örtüsü hakkında ilk kez bilgi verilmektedir. Çam ormanlarında bir oligofag yaprak zararlısı olan Doğu Çam Kese Güvesi'nin (Thaumetopoea wilkinsoni Tams, 1925), araştırma alanında yüksek popülasyon yoğunluğuna sahip olduğu gözlenmiştir.

Anahtar kelimeler: Lepidoptera, Kelebek, Güve, Fauna, Muğla, Türkiye 


\section{Introduction}

Muğla province is located in south-western Anatolia, on the Aegean Sea, borders with Antalya, Aydın, Burdur, Denizli provinces, and the Greek islands face the coast of the province. The city is well known for Turkey's largest holiday resorts, such as Bodrum, Marmaris and Fethiye. Although investigations on Lepidoptera fauna in Muğla province have provided contributions in recent years, studies are still not sufficient and it has not been determined to reveal exactly.

Published reports on the butterflies and moths (Lepidoptera) from Muğla province include: Hacker et al. (1986) contributed to noctuid moth fauna of Turkey and, gave records of two species from Muğla. Lastuvka et al. (1990 [1989]); van Oorschot \& van den Brink (1992); Hensle (1993) published a few Lepidoptera specimens collected from the province. De Bros (1991) presented results of his expedition to Antalya province, and mentioned some species collected from Muğla province. Baisch et al. (1998) recorded thirteen Noctuoidea species. Mol et al. (2003) listed Lepidoptera species of Butterfly Valley and reported twenty-four moth species in Arctiidae, Geometridae, Lasiocampidae, Lymantriidae, Noctuidae, Notodontidae, Pyralidae, Sphingidae and Zygaenidae families. Baron (2014) published a list of 168 species of Noctuoidea, Bombycoidea, Lasiocampoidea, Drepanoidea and Axioidea. Koçak \& Kemal (2018) gave about 260 Macro- and Micro-Lepidoptera species with a checklist from Turkey. Baron (2019) presented in total 299 moth species from Muğla province with two new records for the Turkish fauna.

The present paper includes a faunistic list of 133 species collected from Muğla, Akyaka Forest Camp. Among them, 31 species are new reported from the province. However, detected plant taxa in the area are given below for the first time.

Akyaka Forest Camp, which has homogeneous vegetation, covers an area of approximately 400 decares. Pinus brutia, Quercus aucheri, Styrax officinalis, Phillyrea latifolia, Pistacia terebinthus, Acacia cynaophylla are the most intense plant species in the region, where consists mostly natural areas and hosts many native and cultivated plant species (Figure 1).

Naturally occurring plants: Pinus brutia, P. pinea, Quercus aucheri, Styrax officinalis, Pistacia atlantica, P. terebinthus, $P$. lentiscus, Olea europaea, Laurus nobilis, Ceratonia, Arbutus andrachne, A. unedo, Vitex agnuscastus, Rhus corrida, Phillyrea latifolia, Ficus carica, Capparis spinoza, Ptilostemon chamaepeuce, Cistus creticus, Vaccinium myrtillus, Smilax aspera, S. excels, Ruseus aculeatus, Paliurus spina-christi, Drimia maritima, Asphodelus, Allium, Arum macculatum, Medicago, Verbascum, Asparagus acutifolius, Prospero autumnale, Narcissus serotinus, Carlina gummifera, Sphiranthes spiralis, Serapias, Ophrys apifera, Limodorum abortivum, Leontondon, Bellis perennis, Anthemis, Matricaria, Salicornia europaea, Osyris alba, Oxalis, Euphorbia, Phlomis, Limonium angustifolium, Carlina corymbosa, Lactuca serriola, Rumex pulcher, Oryzopsis miliacea.

Cultivated plants: Platanus orientalis, Liquidambar orientalis, Acacia cynaophylla, Acacia saligna, Acacia sp., Nerium oleander, Robinia pseudoacacia, Chamaerops humilis, Agave americana, Eucalyptus, Ailanthus glandolusa, Cupressus sempervirens, Juniperus foetidissima, Iris germenica, Mirabilis jalapa, Pyrus, Punica granatum, Prunus persica, Canna, Rubus, Lantana camara, Convolvulus, Ipomoea purpurea, I. indica, I. batatas, Senecio tamoides, Ligustrum vulgare, Eriobotrya japonica. 


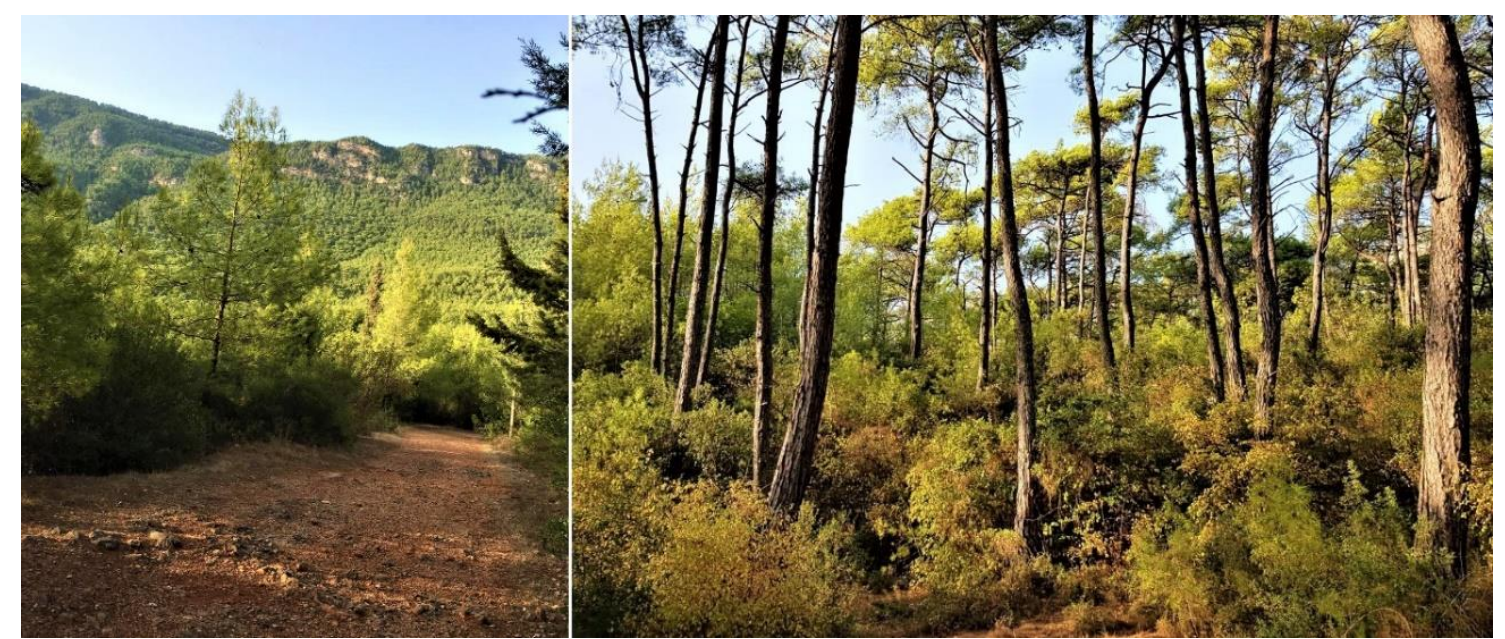

Figure 1. Habitat of Akyaka Forest Camp, Gökova, Ula district, Muğla prov., south-western Turkey, 14.09.2020

\section{Material and Methods}

The materials were collected and photographed from Muğla prov., Ula district, Gökova, Akyaka Forest Camp in 2018-2020 by second author. The specimens coming to the light were caught and packed. Then, the materials were pinned, mounted, labelled, and deposited according to standard entomological methods. External characters of stretched specimens were photographed with a Fujifilm Finepix HS30 EXR digital camera. Preparations of some specimens genitalia were carried out for exact diagnosis, embedded as permanent slides in Euparal under a Nikon SMZ1000 stereomicroscope. The following sources utilized for diagnosis and systematics: Hacker, 1985a; 1985b; 1986; de Freina \& Witt, 1987; 1990; 2001; Fibiger, 1990; 1993; 1997a, 1997b; Ronkay \& Fabian, 1990; Ronkay \& Ronkay, 1994; 1995; Hesselbarth et al., 1995; Kuhna \& Schmitz, 1997; Hausmann, 2001; 2004; Ronkay et al., 2001; Goater et al., 2003; Mironov, 2003; Zilli et al., 2005; Fibiger \& Hacker, 2007; Slamka, 2008; 2011; 2013; Fibiger et al., 2009; 2010; Leraut, 2009; 2014; Witt \& Ronkay, 2011; Hausmann \& Viidalepp, 2012; Skou \& Sihvonen, 2015; Kemal \& Koçak, 2015; 2018; Müller et al., 2019. Specimens are deposited in the collection of Batman University, Faculty of Science and Arts, Department of Biology, Entomology laboratory, Batman, Turkey.

\section{Abbreviations}

Papi: Papilionoidea, Geo: Geometroidea, Cos: Cossoidea, Lasio: Lasiocampoidea, Bomy: Bombycoidea, Drep: Drepanoidea, Noct: Noctuoidea, Pyr: Pyraloidea.

\section{Results}

List of Butterflies (29 Species)

Superfamily Papilionoidea Latreille, 1802

Family Lycaenidae [Leach], [1815]

Glaucopsyche alexis (Poda, 1761)

Lycaena phlaeas (Linnaeus, 1761)

Polyommatus loewii (Zeller, 1847)

Polyommatus daphnis ([Denis \&

Schiffermüller], 1775)

Polyommatus icarus (Rottemburg, 1775)

Satyrium acaciae (Fabricius, 1787)

Tarucus balkanicus (Freyer, [1843])

Family Nymphalidae Rafinesque, 1815 Argynnis pandora ([Denis \& Schiffermüller], 1775)

Charaxes jasius (Linnaeus, 1767)

Danaus chrysippus (Linnaeus, 1758)

Vanessa cardui (Linnaeus, 1758)

Vanessa atalanta (Linnaeus, 1758)

Subfamily Satyrinae Boisduval, 1833

Hipparchia fatua (Freyer, 1844)

Hipparchia syriaca (Staudinger, 1871)

Hyponephele lycaon (Rottemburg, 1775)

Kirinia roxelana (Cramer, [1777])

Pararge aegeria (Linnaeus, 1758) 
Family Papilionidae Latreille, [1802]

Iphiclides podalirius (Linnaeus, 1758)

Papilio machaon Linnaeus, 1758

Family Pieridae Duponchel, [1835]

Colias crocea (Fourcroy, 1785)

Colias aurorina Herrich-Schäffer, [1850]

Pieris ergane (Geyer, [1828])

Pieris rapae (Linnaeus, 1758)

Pieris brassicae (Linnaeus, 1758)

Family Hesperiidae Latreille, 1809

Erynnis marloyi (Boisduval, [1834])

Gegenes pumilio (Hoffmannsegg, 1804)

Ochlodes sylvanus (Esper, [1777])

Spialia orbifer (Hübner, [1823])

Thymelicus lineolus (Ochsenheimer, 1808)

List of Moths (104 Species)

Superfamily Geometroidea Leach, 1815

Family Geometridae Leach, 1815

Ascotis selenaria ([Denis \& Schiffermüller], 1775)

Aspitates ochrearia (Rossi, 1794) (Fig. 2a)

Camptogramma bilineata (Linnaeus, 1758)

Chiasmia aestimaria (Hübner, [1809])

Cyclophora puppillaria (Hübner, [1799])

Epirrhoe molluginata (Hübner, [1813])

Eucrostes indigenata (De Villers, 1789)

Gnophos sartata Treitschke, 1827

Gymnoscelis rufifasciata (Haworth, [1809])

Hylaea fasciaria (Linnaeus, 1758)

Idaea degeneraria (Hübner, [1799])

Idaea elongaria (Rambur, 1833)

Idaea filicata (Hübner, [1799])

Idaea obsoletaria (Rambur, 1833)

Idaea sylvestraria (Hübner, [1799])

Idaea trigeminata (Haworth, [1809])

Isturgia arenacearia ([Denis \&

Schiffermüller], 1775) (Fig. 2b)

Isturgia murinaria ([Denis \& Schiffermüller], 1775)

Chlorissa viridata (Linnaeus, 1758)

Nychiodes waltheri Wagner, 1919

Peribatodes rhomboidaria ([Denis \&

Schiffermüller], 1775)

Peribatodes umbraria (Hübner, [1809])

Problepsis ocellata (Frivaldszky, 1845) (Fig.

2c)

Rhodometra sacraria (Linnaeus, 1767)

Scopula imitaria (Hübner, [1799]) (Fig. 2d)

Scopula marginepunctata (Goeze, 1781)

Scopula submutata (Treitschke, 1828)
Stueningia taurica Hausmann, 1993

Superfamily Cossoidea Leach, [1815]

Family Cossidae Leach, [1815]

Dyspessa ulula (Borkhausen, 1790)

Zeuzera pyrina (Linnaeus, 1761)

Superfamily Lasiocampoidea Harris, 1841

Family Lasiocampidae Harris, 1841

Malacosoma castrensis (Linnaeus, 1758)

Phylodesma tremulifolia (Hübner, [1810])

Superfamily Bombycoidea Latreille, 1802

Family Saturniidae Boisduval, 1837

Saturnia pyri ([Denis \& Schiffermüller], 1775)

Family Sphingidae Latreille, [1802]

Daphnis nerii (Linnaeus, 1758)

Hyles euphorbiae (Linnaeus, 1758)

Hyles livornica (Esper, [1780])

Macroglossum stellatarum (Linnaeus, 1758)

Sphinx pinastri Linnaeus, 1758 (Fig. 2e)

Superfamily Drepanoidea Boisduval, 1828

Family Drepanidae Boisduval, 1828

Watsonalla uncinula (Borkhausen, 1790)

Superfamily Noctuoidea Latreille, 1809

Family Thaumetopoeidae Aurivillius, 1891

Thaumetopoea wilkinsoni Tams, 1925 (Fig.

2f)

Family Erebidae Leach, [1815]

Calymma communimacula ([Denis \&

Schiffermüller], 1775)

Catocala conversa (Esper, 1783)

Catocala elocata (Esper, [1787])

Catocala hymenaea ([Denis \&

Schiffermüller], 1775)

Catocala nupta (Linnaeus, 1767)

Catocala nymphagoga (Esper, [1787])

Chrysodeixis chalcites (Esper, [1789])

Dysgonia algira (Linnaeus, 1767)

Eublemma ostrina (Hübner, [1808])

Eublemma parva (Hübner, [1808])

Grammodes bifasciata (Petagna, 1787)

Grammodes stolida (Fabricius, 1775)

Metachrostis velox (Hübner, [1813])

Ophiusa tirhaca (Cramer, [1777])

Plecoptera inquinata (Lederer, 1857)

Polypogon plumigeralis (Hübner, [1825])

(Fig. 2g) 
Subfamily Hypenodinae Forbes, 1954 Micronoctua karsholti (Fibiger, 1997)

Subfamily Arctiinae Leach, [1815]

Dysauxes famula (Freyer, 1836)

Eilema caniolum (Hübner, [1808])

Eilema costalis (Zeller, 1847)

Euplagia quadripunctaria (Poda, 1761)

Utetheisa pulchella (Linnaeus, 1758)

Family Noctuidae Latreille, 1809

Agrotis bigramma (Esper, [1790])

Agrotis puta (Hübner, [1803])

Agrotis segetum ([Denis \& Schiffermüller], 1775)

Aporophyla canescens (Duponchel, 1826)

Aporophyla nigra (Haworth, [1809])

Autographa gamma (Linnaeus, 1758)

Bryophila rectilinea (Warren, 1909)

Caradrina clavipalpis (Scopoli, 1763)

Caradrina levantina Hacker, 2004

Chersotis rectangula (Fabricius, 1787)

Cryphia ochsi (Boursin,1941)

Dicycla oo (Linnaeus, 1758)

Divaena haywardi (Tams, 1926) (Fig. 2h)

Egira tibori Hreblay, 1994

Haemerosia renalis (Hübner, [1813])

Helicoverpa armigera (Hübner, [1808])

Heliothis nubigera Herrich-Schäffer, [1851]

Heliothis peltigera ([Denis \& Schiffermüller], 1775)

Hoplodrina ambigua ([Denis \&

Schiffermüller], 1775)

Mormo maura (Linnaeus, 1758)

Mythimna vitellina (Hübner, [1808])

Mythimna albipuncta ([Denis \&

Schiffermüller], 1775)

Noctua pronuba (Linnaeus, 1758)
Noctua janthina ([Denis \& Schiffermüller], 1775)

Polymixis serpentina (Treitschke, 1825) (Fig.

2k)

Trichoplusia ni (Hübner, [1803])

Sesamia cretica Lederer, 1857

Spodoptera cilium Guenée, 1852

Spodoptera exigua (Hübner, [1808])

Spodoptera littoralis (Boisduval, 1833)

Superfamily Pyraloidea Latreille, 1809

Family Pyralidae Latreille, 1809

Endotricha flammealis ([Denis \& Schiffermüller], 1775)

Pyralis farinalis (Linnaeus, 1758)

Family Crambidae Latreille, 1810

Antigastra catalaunalis (Duponchel, 1833)

Duponchelia fovealis Zeller, 1847

Hellula undalis (Fabricius, 1781)

Herpetogramma licarsisalis (Walker, 1859)

Hydriris ornatalis (Duponchel, 1832) (Fig. 21)

Palpita vitrealis (Rossi, 1794)

Paratalanta hyalinalis (Hübner, 1796)

Pyrausta pauperalis (Staudinger, 1879)

Spoladea recurvalis (Fabricius, 1775) (Fig. $2 \mathrm{~m})$

Udea ferrugalis (Hübner, 1796)

\section{Discussion}

As a result of the researches conducted in Muğla province, 29 butterfly and 104 moth species were identified in the superfamilies Papilionoidea, Geometroidea, Cossoidea, Lasiocampoidea, Bombycoidea, Drepanoidea, Noctuoidea, Pyraloidea and, 31 of them are new recorded (Koçak \& Kemal, 2018; Baron, 2019) (Table 1).

Table 1. Determined species and new record numbers in superfamilies

\begin{tabular}{|c|c|c|c|c|c|c|c|c|c|}
\hline & \multirow{2}{*}{$\begin{array}{c}\text { Butterflies } \\
\text { Papi }\end{array}$} & \multicolumn{8}{|c|}{ Moths } \\
\hline & & Geo & Cos & Lasio & Bomy & Drep & Noct & Pyr & Total \\
\hline Determined species & 29 & 28 & 2 & 2 & 6 & 1 & 53 & 12 & 133 \\
\hline New record & 2 & 16 & - & - & - & 1 & 5 & 7 & 31 \\
\hline
\end{tabular}




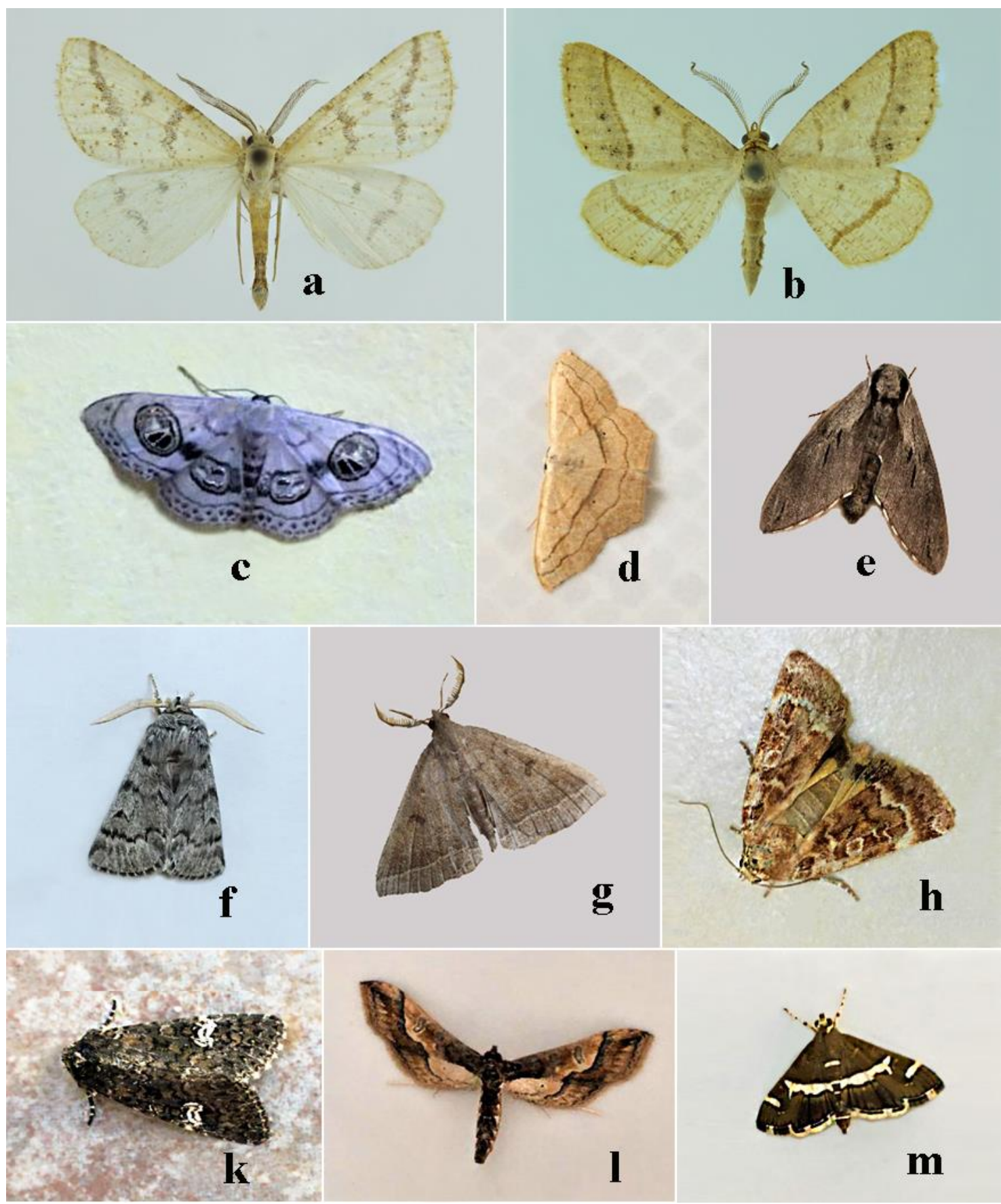

Figure 2. Natural postures and stretched of some adult species: a. Aspitates ochrearia, b. Isturgia arenacearia, c. Problepsis ocellata, d. Scopula imitaria, e. Sphinx pinastri, f. Thaumetopoea wilkinsoni, g. Polypogon plumigeralis, h. Noctua haywardi, k. Polymixis serpentina, 1. Hydriris ornatalis, m. Spoladea recurvalis

The new discovered two butterfly and twenty-nine moth species are as follows according to the families:

Pieridae: Pieris ergane. Hesperiidae: Ochlodes sylvanus. - Geometridae: Ascotis selenaria, Aspitates ochrearia, Epirrhoe molluginata, Gnophos sartata, Hylaea fasciaria, Idaea elongaria, I. obsoletaria, I. trigeminata, Isturgia arenacearia, I. murinaria, Chlorissa viridata, Nychiodes waltheri, Peribatodes umbraria, Scopula marginepunctata, S. submutata, Stueningia taurica. Drepanidae: Watsonalla uncinula. Erebidae: Calymma communimacula, Grammodes bifasciata. Noctuidae: Chersotis rectangular, Dicycla oo, Haemerosia renalis. 
Crambidae: Antigastra catalaunalis, Hellula undalis, Herpetogramma licarsisalis, Hydriris ornatalis, Paratalanta hyalinalis, Pyrausta pauperalis, Spoladea recurvalis.

Although there have been investigations on Noctuoidea and Bombycoidea in Muğla province, studies on Geometroidea and Pyraloidea were insufficient. Therefore, most new records have been in these groups. The highest number of species are determined from Papilionoidea and Geometroidea superfamilies (Table 1). However, there is no information on the plants of the study area, Akyaka Forest Camp, in this research detected 52 natural and 28 cultivated plant taxa (see introduction) are also mentioned.

Some Lepidoptera caterpillars are of major pests and have economic importance (Akınc1 \& Kurdoğlu, 2019). Thaumetopoea wilkinsoni Tams, 1925, which is an oligofag defoliator in pine forests, develops on native and introduced Pinus spp. in the East Mediterranean, from western Turkey to Israel. In Turkey, mainly colonized on $P$. nigra, $P$. brutia brutia, $P$. halepensis, $P$. pinea, and $P$. radiata (Çanakçığlu and Mol, 1998). The species is detected with high population density in the study area.

\section{Ethics Committee Approval N/A}

\section{Peer-review}

Externally peer-reviewed.

\section{Author Contributions}

Conceptualization: E.S.; Investigation: E.S., N.Y.; Material and Methodology: E.S., N.Y.; Supervision: E.S., N.Y.; Visualization: E.S., N.Y.; Writing-Original Draft: E.S.; Writing-review\&Editing: E.S.; Other: E.S., N.Y. All authors have read and agreed to the published version of manuscript.

\section{Conflict of Interest}

The authors have no conflicts of interest to declare.

\section{Funding}

The authors declared that this study has received no financial support.

\section{Acknowledgements}

We thank Dr. Kesran Akın (University of Bitlis Eren, Turkey) for helping on the diagnosis of pyralid moths. We are grateful to Turgut Yılmaz, Ragıp Şentürk and Rasim Çetiner (Turkey) for identification of the plant species.

\section{References}

Baisch G., Beshkov S., Gelbrecht J., Hacker H., Huber K., Kallies A., Kautt P., Löbel H., Lehmann L. \& Petersen M. (1998). Bausteine zur Fauna der Noctuoidea der Türkei. Esperiana, 6, 213-373.

Baron T. (2014). Collection Records of Noctuoidea and Bombycoidea (Lepidoptera) from a Location near Marmaris in SouthWestern Anatolia, Turkey. Esperiana, 19, 263-296.

Baron T. (2019). A Checklist for the Superfamilies Noctuoidea, Bombycoidea, Lasiocampoidea, Drepanoidea, Axioidea, Zygaenoidea and Cossoidea (Lepidoptera) of the Turkish province of Muğla in south-western Anatolia, Turkey. Entomofauna, 40(2), 325-377.

Çanakçıŏlu H. \& Mol T. (1998). Orman Entomolojisi Zararlı ve Yararlı Böcekler. Istanbul Üniversitesi Orman Ekolojisi, 1-541.

De Bros E. (1991). Beitrag zur Kenntnis der Lepidopteren-Fauna von Antalya und des lykischen Taurus in der Südwesttürkei. Mitteilungen der Münchner Entomologischen Basel, 41(4), 112-135.

De Freina J. \& Witt T.J. (1987). Die Bombyces und Sphinges der Westpalaearktis. Band 1. München.

De Freina J. \& Witt T.J. (1990). Die Bombyces und Sphinges der Westpalaearktis. Band 2. München.

De Freina J. \& Witt T.J. (2001). Die Bombyces und Sphinges der Westpalaearktis. Band 3. München.

Fibiger M. \& Hacker H. (2007). Noctuidae Europaeae, Vol. 9, Amphipyrinae, Condicinae, Eriopinae, Xyleninae (part). Sorø.

Fibiger M. (1990). Noctuidae Europaeae, v.1, Noctuinae I. Sorø.

Fibiger M. (1993). Noctuidae Europaeae, v.2, Noctuinae II. Sorø.

Fibiger M. (1997a). Noctuidae Europaeae, v.3, Noctuinae III. Sorø.

Fibiger M. (1997b). Micronoctua karsholti n.gen. et n.sp.: An astonishingly small noctuid moth (Noctuidae). Nota Lepidopterologica, 20, 2330.

Fibiger M., Ronkay L., Steiner A. \& Zilli A. (2009). Noctuidae Europaeae, v.11, 
Pantheinae, Dilobinae, Acronictinae, Eustrotiinae, Nolinae, Bagisarinae, Acontiinae, Metoponiinae, Heliothinae and Bryophilinae. Sorø.

Fibiger M., Ronkay L., Yela J. L. \& Zilli A. (2010). Noctuidae Europaeae, v.12, Rivulinae, Boletobiinae, Hypenodinae, Araeopteroniniae, Eublemminae, Herminiinae, Hypeninae, Phytometrinae, Euteliinae and Micronoctuinae. Sorø.

Goater B., Ronkay L. \& Fibiger M. (2003). Noctuidae Europaeae, v.10, Catocalinae, Plusiinae. Sorø.

Hacker H. (1985a). Erster Beitrag zur systematischen Erfassung der Noctuidae der Türkei. Atalanta, 17, 1-25.

Hacker H. (1985b). Dritter Beitrag zur Erfassung der Noctuiden der Türkei. Neue Entomologische Nachrichten, 15, 1-67.

Hacker H. (1986). 2. Beitrag zur Erfassung der Noctuidae der Türkei. Spixiana, 9, 25-81.

Hacker H., Kuhna, P. \& Gross F. J. (1986). 4. Beitrag zur Erfassung der Noctuidae der Türkei. Mitteilungen der Münchner Entomologischen Gesellschaft, 76, 79-141.

Hausmann A. \& Viidalepp J. (2012). The Geometrid Moths of Europe. v.3, Larentiinae I. Apollo Books, Stenstrup.

Hausmann A. (2001). The Geometrid Moths of Europe Vol. 1. Apollo Books, Stenstrup.

Hausmann A. (2004). The Geometrid Moths of Europe, v.2, Sterrhinae. Apollo Books, Stenstrup.

Hensle J. (1993). Beobachtungen bei westanatolischen Osterluzeifaltern (Lepidoptera: Papilionidae). Nachrichten entomologische Vereins Apollo Frankfurt/Main, 14, 289-299.

Hesselbarth G., van Oorschot H. \& Wagener S. (1995). Die Tagfalter der Türkei unter Berücksichtigung der angrenzenden Länder. Bocholt.

Kemal M. \& Koçak A.Ö. (2015). Notes on two species of Geometridae from Hatay Province (S. Turkey) (Lepidoptera). Cesa News, 119, 16.

Kemal M. \& Koçak A. Ö. (2018). On the Stueningia species in Turkey (Lepidoptera, Geometridae). Cesa News, 151, 1-3.

Koçak A. Ö. \& Kemal M. (2018). A synonymous and distributional list of the species of the Lepidoptera of Turkey. Centre for Entomological Studies, Memoirs, 8, 1-487.

Kuhna P. \& Schmitz B. (1997). Eine neue Art der Gattung Valeria aus der Süd-Türkei (Lepidoptera, Noctuidae). Jahresberichte des Naturwissenschaftlichen Vereins in Wuppertal, 50, 94-99.
Akıncı H. A. \& Kurdoğlu O. (2019). Damage Level of Cydalima perspectalis (Lepidoptera: Crambidae) on Naturally Growing and Ornamental Box Populations in Artvin, Turkey. Kastamonu Univ., Journal of Forestry Faculty, 19(2), 144-151.

Lastuvka Z., Malicky H., Hüttinger E., Rausch H. \& Ressl F. (1990 [1989]). Sesien-Funde aus Europa und dem Mediterrangebiet (Lepidoptera, Sesiidae). Zeitschrift der Arbeitsgemeinschaft Österreichischer Entomologen, 41, 105-110.

Leraut P. (2009). Moths of Europe. v.2, Geometrid moths. N.A.P. ed., Verrières-le-Buisson.

Leraut P. (2014). Moths of Europe. Volume 4 Pyralids 2. N.A.P. ed., Verrières-le-Buisson.

Mironov V. (2003). The Geometrid Moths of Europe, v.4, Larentiinae II. Apollo Books, Stenstrup.

Mol T., Avci M. \& Dutkuner I. (2003). Fethiye Kelebekler Vadisi Florasi ve Lepidoptera Faunas1. Istanbul Üniversitesi Orman Fakultesi 53, 15-24.

Müller B., Erlacher S., Hausmann A., Rajaei H., Sihvonen P. \& Skou P. (2019). Geometrid Moths of Europe, v.6, Ennominae II. Leiden, Brill.

Van Oorschot H. \& van den Brink H. (1992). Eight years of systematic investigation of the Rhopalocera of Turkey (Lepidoptera: Satyridae, Lycaenidae). Nota Lepidopterologica Supplement, 3, 82-89.

Ronkay G. \& Ronkay L. (1994). Noctuidae Europaeae, v.6, Cuculliinae I. Sorø.

Ronkay G. \& Ronkay L. (1995). Noctuidae Europaeae, v.7, Cuculliinae II. Sorø.

Ronkay L. \& Fabian G. Y. (1990). Contributions to the Noctuidae fauna of Turkey. Annales Historico-Naturales Musei Nationalis Hungarici, 81, 115-123.

Ronkay L., Yela J. L. \& Hreblay M. (2001). Noctuidae Europaeae, v.5, Hadeninae II. Sorø.

Skou P. \& Sihvonen P. (2015). Subfamily Ennominae I, v.5, [in] Hausmann, A. (ed.), The geometrid moths of Europe 5. Brill, Leiden.

Slamka F. (2008). Pyraloidea of Europe (Lepidoptera) Volume 2. Crambinae \& Schoenobiinae. Identification, Distribution, Habitat, Biologie. Bratislava.

Slamka F. (2011). Pyraloidea of Europe (Lepidoptera) v.1. Pyralinae, Galleriinae, Epipaschiinae, Cathariinae \& Odontiinae. Identification, Distribution, Habitat, Biologie. Bratislava.

Slamka F. (2013). Pyraloidea of Europe (Lepidoptera), v.3, Pyraustinae \& Spilomelinae. Identification, Distribution, Habitat, Biologie. Bratislava. 
Witt T.J. \& Ronkay L. (2011). Noctuidae Europaeae, v.13, Lymantriinae and Arctiinae. Sorø.
Zilli A., Ronkay, L. \& Fibiger M. (2005). Noctuidae Europaeae, v.8, Apameini. Sorø. 